\title{
Magnetic Diagram of the High-Pressure Stabilized Multiferroic Perovskites of the $\mathrm{BiFe}_{1-y} \mathrm{Sc}_{y} \mathrm{O}_{3}$ Series
}

\author{
Elena L. Fertman ${ }^{1}\left(\mathbb{D}\right.$, Alexey V. Fedorchenko ${ }^{1}{ }^{\circledR}$, Erik Čižmár ${ }^{2}{ }^{\circledR}$, Serhii Vorobiov $^{2}{ }^{\circledR}$, \\ Alexander Feher ${ }^{2}$, Yury V. Radyush ${ }^{3}$, Anatoli V. Pushkarev ${ }^{3}$, Nikolai M. Olekhnovich ${ }^{3}$, \\ Andrius Stanulis ${ }^{4}$, Andrew R. Barron ${ }^{4,5,6}$, Dmitry D. Khalyavin ${ }^{7, *}$ and Andrei N. Salak ${ }^{8, *}$ (i) \\ 1 B. Verkin Institute for Low Temperature Physics and Engineering of the National Academy of Sciences \\ of Ukraine (NASU), Nauky 47, 61103 Kharkiv, Ukraine; fertman@ilt.kharkov.ua (E.L.F.); \\ fedorchenko.alexey@gmail.com (A.V.F.) \\ 2 Faculty of Sciences, Institute of Physics, P. J. Šafárik University, Park Angelinum 9, 04154 Košice, Slovakia; \\ erik.cizmar@upjs.sk (E.Č.); serhii.vorobiov@upjs.sk (S.V.); alexander.feher@upjs.sk (A.F.) \\ 3 Scientific-Practical Materials Research Centre of the National Academy of Sciences of Belarus (NASB), \\ P. Brovka 19, 220072 Minsk, Belarus; radyush@ifttp.bas-net.by (Y.V.R.); pushk@physics.by (A.V.P.); \\ olekhnov@ifttp.bas-net.by (N.M.O.) \\ 4 Energy Safety Research Institute (ESRI), Bay Campus, Swansea University, Fabian Way, \\ Swansea SA1 8EN, UK; Andrius.stanulis@swansea.ac.uk (A.S.); a.r.barron@swansea.ac.uk (A.R.B.) \\ 5 Department of Chemistry and Department of Materials Science and Nanoengineering, Rice University, \\ Houston, TX 77005, USA \\ 6 Faculty of Engineering, Universiti Teknologi Brunei, Jalan Tungku Link, Gadong, \\ Bandar Seri Begawan BE1410, Brunei \\ 7 ISIS Facility, Rutherford Appleton Laboratory, Chilton, Didcot, Oxfordshire OX11 0QX, UK \\ 8 Department of Materials and Ceramics Engineering/CICECO-Aveiro Institute of Materials, \\ University of 3810-193 Aveiro, Portugal \\ * Correspondence: dmitry.khalyavin@stfc.ac.uk (D.D.K.); salak@ua.pt (A.N.S.)
}

Received: 30 September 2020; Accepted: 16 October 2020; Published: 17 October 2020

check for updates

\begin{abstract}
Magnetic properties of the high-pressure stabilized perovskite $\mathrm{BiFe}_{1-y} \mathrm{Sc}_{y} \mathrm{O}_{3}$ phases $(0.1 \leq y \leq 0.9)$ have been studied by means of magnetization measurements and neutron diffraction. The metastable perovskites of this series undergo irreversible polymorphic transformations upon annealing, the phenomenon referred to as conversion polymorphism. It has been found that the solid solutions with $y \geq 0.70$ exhibit no long-range magnetic ordering regardless of their polymorph modification, while those with $y \leq 0.60$ are all antiferromagnets. Depending on the scandium content, temperature and structural distortions, three types of the antiferromagnetic orderings, involving collinear, canted and cycloidal spin arrangements, have been revealed in the phases obtained via conversion polymorphism and the corresponding magnetic phase diagram has been suggested.
\end{abstract}

Keywords: high-pressure synthesis; conversion polymorphism; oxygen octahedral tilting; G-type antiferromagnetic; weak ferromagnetism; collinear magnetic ground state

\section{Introduction}

Chemical modifications of one of the most known type-I multiferroics, bismuth ferrite [1], make it possible to tune the properties of the parent composition (e.g., to adjust the ranges of the antiferromagnetic and the ferroelectric phase transitions) and to obtain new crystalline and magnetic structures [2,3]. While bulk single-phase perovskites with considerable and even complete isovalent substitutions of bismuth in $\mathrm{BiFeO}_{3}$ can be easy produced using the conventional ceramic routes [4-6], 
the formation of the compositions with more than 15 mol.\% substitution of iron (except for $\mathrm{Mn}^{3+}$ substituted $\mathrm{BiFeO}_{3}$ where up to $30 \mathrm{~mol} . \%$ can be achieved [7]) requires a high-pressure synthesis.

Using the high-pressure synthesis, the $\mathrm{Bi}\left(\mathrm{Fe}, B^{3+}\right) \mathrm{O}_{3}$ solid solutions with the $\mathrm{Fe}^{3+}$-to- $B^{3+}$ substitution of 50 mol.\% and above $\left(B^{3+}=\mathrm{Mn}[8,9]\right.$, Co [10-12], Cr [13,14], Ga [15,16]) have been obtained. The composition-driven sequences of the crystalline phases and their structural distortions as well as the feasibility of their application as materials with desired functionalities (in particular, as multiferroics and piezoelectrics) were studied $[10,17]$. Even though the magnetic behavior of particular compositions of those solid solution systems were investigated in great detail, only a few magnetic phase diagrams have been published so far $[8,16]$.

We have recently prepared and characterized the entire series of the high-pressure stabilized perovskite solid solutions of the $\mathrm{BiFeO}_{3}-\mathrm{BiScO}_{3}$ system $[18,19]$. $\mathrm{BiScO}_{3}$ was chosen as the end member since substitution of $\mathrm{Fe}^{3+}$ by a considerably bigger $\mathrm{Sc}^{3+}$ was expected to induce new crystal structures and thereby new combinations of multiferroic properties. Indeed, three structure fields with the rhombohedral $R 3 c$ symmetry and the $\sqrt{2} a_{p} \times \sqrt{ } 2 a_{p} \times 2 \sqrt{ } 3 a_{p}$ superstructure (where $a_{p}$ is the pseudocubic perovskite unit-cell parameter), the orthorhombic Pnma symmetry $\left(\sqrt{2} a_{p} \times 4 a_{p} \times 2 \sqrt{ } 2 a_{p}\right)$ and the monoclinic $C 2 / c$ one $\left(\sqrt{ } 6 a_{p} \times \sqrt{ } 2 a_{p} \times \sqrt{ } 6 a_{p}\right)$ were revealed [18]. Moreover, in the $\mathrm{BiFe}_{1-y} \mathrm{Sc}_{y} \mathrm{O}_{3}$ series, the phenomenon of annealed-stimulated irreversible transformations between metastable perovskite phases (conversion polymorphism) was observed. We have revealed at least three compositional ranges in this solid solution system, where the post-synthesis annealing results in the formation of different perovskite polymorphs with new combinations of structural distortions and magnetic orders [19].

While the as-prepared (non-annealed) phase of the $\mathrm{BiFe}_{0.50} \mathrm{Sc}_{0.50} \mathrm{O}_{3}$ composition is the antipolar Pnma, the conversion-stabilized modification is a polar orthorhombic with the Ima2 symmetry $\left(2 a_{p} \times \sqrt{ } 2 a_{p} \times \sqrt{ } 2 a_{p}\right)$ [20]. Both the polar and the antipolar polymorphs of $\mathrm{BiFe}_{0.5} \mathrm{Sc}_{0.5} \mathrm{O}_{3}$ are G-type antiferromagnets (AFM) with a weak ferromagnetic (FM) component below the Néel temperature, $T_{\mathrm{N}} \sim 220 \mathrm{~K}$. Although the type of the magnetic order and the $T_{\mathrm{N}}$ value are the same for both polymorphs, magnetization of the polar polymorph below $T_{\mathrm{N}}$ was found to be 2-5 times smaller than that of the antipolar one [20]. Besides, the $\mathrm{BiFe}_{0.50} \mathrm{Sc}_{0.50} \mathrm{O}_{3}$ polymorphs were shown to be also rather different in terms of effects caused by their magnetic anisotropy [21,22]. In the range of the $\mathrm{BiFe}_{1-y} \mathrm{Sc}_{y} \mathrm{O}_{3}$ compositions with $y$ close to 0.30, annealing of the as-prepared antipolar Pnma phase leads to irreversible transformation into a rhombohedral $R 3 c$ polymorph with a very unusual collinear magnetic ground state [19]. The monoclinic $\mathrm{C2} / \mathrm{c}$ phase of the as-prepared $\mathrm{BiFe}_{1-y} \mathrm{Sc}_{y} \mathrm{O}_{3}$ compositions with $y \geq 0.7$ irreversibly transforms into a new antiferroelectric-like phase with the orthorhombic Pnma symmetry and the $2 \sqrt{ } 2 a_{p} \times 4 a_{p} \times \sqrt{ } 2 a_{p}$ superstructure [19]. Hereafter, the modifications with the superstructures $\sqrt{ } 2 a_{p} \times 4 a_{p} \times 2 \sqrt{ } 2 a_{p}$ and $2 \sqrt{ } 2 a_{p} \times 4 a_{p} \times \sqrt{ } 2 a_{p}$ are denoted as Pnma (I) and Pnma (II), respectively. It should be stressed here that the different unit cell choice in these modifications implies essentially different distortions involved.

A temperature-composition phase diagram of the $\mathrm{BiFe}_{1-y} \mathrm{Sc}_{y} \mathrm{O}_{3}$ series plotted based on the data of the in situ temperature powder diffraction studies demonstrates a sequence of the perovskite-type structures different in respect of combinations of the oxygen octahedral tiltings [19]. It should be pointed out that the octahedral tilting not only determines the structure distortion (symmetry) but also has an effect on the long-range magnetic order in perovskites. In particular, it has been recently shown that the component of the Dzyaloshinskii-Moriya vector, which couples the G-type AFM and orthogonal FM modes, is imposed by anti-phase octahedral tiltings [20,23].

Here we report the compositional behavior of magnetic properties of the $\mathrm{BiFe}_{1-y} \mathrm{Sc}_{y} \mathrm{O}_{3}$ solid solutions $(0.1 \leq y \leq 0.9)$ before and after annealing that results in the irreversible polymorphic transformations. The obtained magnetic diagram is considered in comparison with the temperature-composition phase diagram of the $\mathrm{BiFeO}_{3}-\mathrm{BiScO}_{3}$ system. 


\section{Materials and Methods}

Ceramics of the $\mathrm{BiFe}_{1-y} \mathrm{Sc}_{y} \mathrm{O}_{3}$ series $(0.1 \leq y \leq 0.9)$ were synthesized under high-pressure from the precursor prepared via either a solid-state reaction or a sol-gel combustion route. Details of the precursor preparation and the high-pressure synthesis can be found in References [18] and [21]. Annealing of the obtained ceramic samples was done in air at $870 \mathrm{~K}$ for $3 \mathrm{~h}$.

Phase analysis of the samples before and after annealing was performed using a PANalytical $X^{\prime}$ Pert Powder X-ray diffractometer (XRD, Ni-filtered $\mathrm{Cu} \mathrm{K} \alpha$ radiation) at room temperature. Before the $X R D$ measurements, the samples were reduced into powders.

Neutron powder diffraction data were collected at the ISIS pulsed neutron and muon facility of the Rutherford Appleton Laboratory (UK) on the WISH diffractometer [24]. The studied samples were loaded into a cylindrical $3 \mathrm{~mm}$ diameter vanadium can and measured in a temperature range of $1.5-300 \mathrm{~K}$. The crystal structure and the magnetic structure of the samples were refined using the FULLPROF package [25].

Magnetic properties of the ceramic samples were measured in the range of 5-300 $\mathrm{K}$ using a commercial Quantum Design MPMS-3 magnetometer in applied fields up to $70 \mathrm{kOe}$ in both zero-field-cooled (ZFC) and field-cooled (FC) modes. High temperature (over the range of 300-700 K) measurements were done using a commercial Quantum Design MPMS-XL magnetometer equipped with oven insert.

\section{Results and Discussion}

Magnetic behavior of the $\mathrm{BiFe}_{1-y} \mathrm{Sc}_{y} \mathrm{O}_{3}$ perovskites with the relative iron content above and below $30 \mathrm{~mol} \%$ (which corresponds to $y<0.70$ and $y \geq 0.70$, respectively) were found to be essentially different.

Regardless of the structure modification, $\mathrm{C} 2 / \mathrm{c}$ or Pnma (II), $\mathrm{BiFe}_{0.3} \mathrm{Sc}_{0.7} \mathrm{O}_{3}$ exhibits no sign of a long-range magnetic ordering down to the low temperatures. It is expectable since the iron content in this compound is below the percolation threshold of $31 \mathrm{~mol} \%(y=0.69)$ for perovskite structures [26], when magnetic $\mathrm{Fe}^{3+}$ still can form a continuous path throughout the crystal to produce a long-range magnetic ordering.

Indeed, the neutron diffraction study has revealed no additional contributions to nuclear reflections that could indicate an onset of magnetic ordering. As an example, Figure 1 demonstrates the results of the crystal structure refinement of the Pnma (II) polymorph of $\mathrm{BiFe}_{0.3} \mathrm{Sc}_{0.7} \mathrm{O}_{3}$ at $1.5 \mathrm{~K}$.

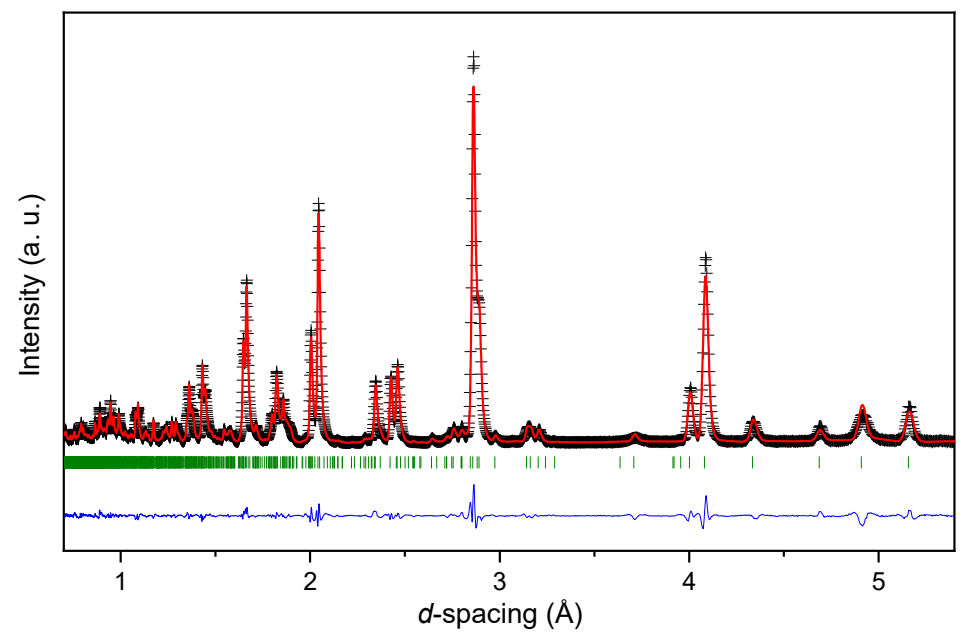

Figure 1. Neutron diffraction pattern of the annealed $\mathrm{BiFe}_{0.30} \mathrm{Sc}_{0.70} \mathrm{O}_{3}$ sample collected at $1.5 \mathrm{~K}$ and refined in the Pnma (II) structural model $\left(\mathrm{R}_{\mathrm{Bragg}}=3.01 \%\right)$. No magnetic ordering was required to achieve the good refinement quality. The cross symbols and solid line (red) represent the experimental and calculated intensities, respectively, and the line below (blue) is the difference between them. Tick marks (green) indicate the positions of Bragg peaks. 
The diffraction data are consistent with the results of the magnetic measurements. The temperature-dependent ZFC and FC curves of $\mathrm{BiFe}_{0.3} \mathrm{Sc}_{0.7} \mathrm{O}_{3}$ coincide down to about $25 \mathrm{~K}$ and slightly diverge below this temperature. The magnetic moment of its monoclinic $\mathrm{C} 2 / \mathrm{c}$ modification as a function of temperature is shown in Figure 2. The Curie-Weiss fitting gives the effective magnetic moment $\mu_{\text {eff }} \sim 2.99 \mu_{\mathrm{B}} / \mathrm{Fe}^{3+}$, which is close to the experimental value for the low-spin $\mathrm{Fe}^{3+}$ in the octahedral environment. Further decrease of the iron content in the $\mathrm{BiFe}_{1-y} \mathrm{Sc}_{y} \mathrm{O}_{3}$ system results in reduction of the paramagnetic response, which vanishes completely in $\mathrm{BiScO}_{3}(y=1)$.
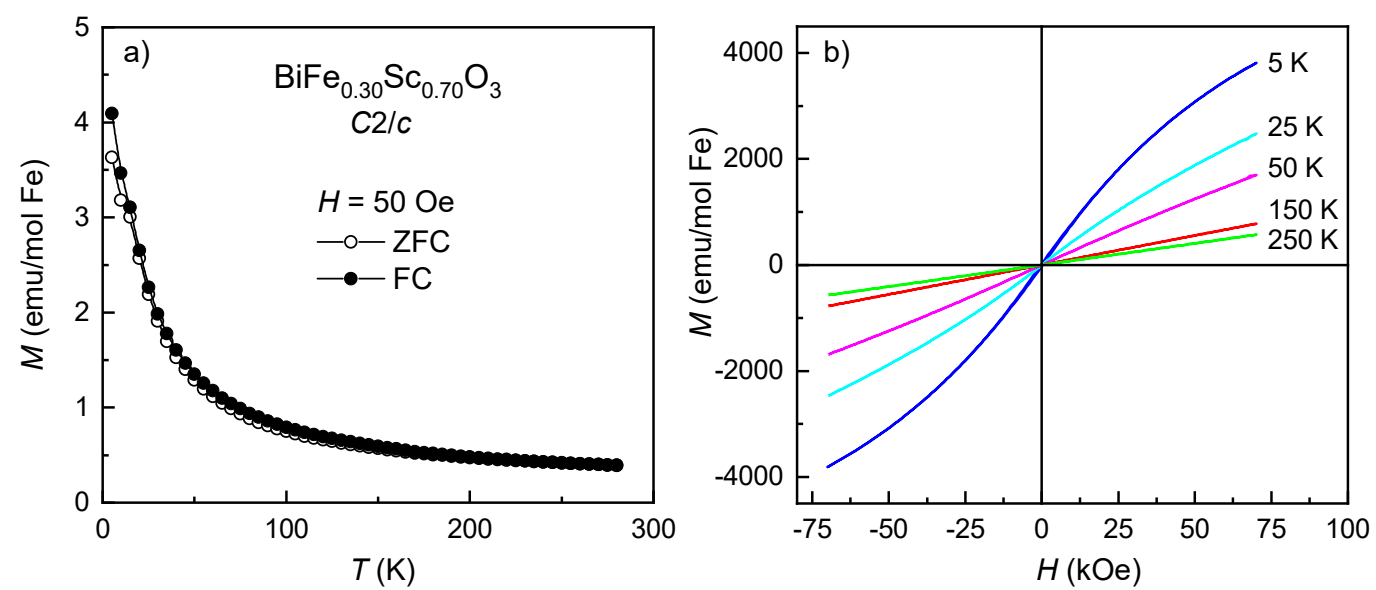

Figure 2. (a) Temperature-dependent $d c$ magnetic moment of the monoclinic $C / 2 c$ (as-prepared) polymorph of $\mathrm{BiFe}_{0.30} \mathrm{Sc}_{0.70} \mathrm{O}_{3}$ measured in the magnetic field of 50 Oe in the zero-field-cooled (ZFC) (open symbols) and field-cooled (FC) (solid symbols) modes; (b) field dependent magnetization loops measured at different temperatures.

The magnetic behavior of the $\mathrm{BiFe}_{1-y} \mathrm{Sc}_{y} \mathrm{O}_{3}$ perovskites with the relative iron content of $60 \mathrm{~mol} . \%$ and above $(y<0.7)$ was revealed to be typical for antiferromagnets. Dilution of the iron magnetic subsystem by scandium leads to the near-linear decrease of the Néel temperature, $T_{\mathrm{N}}$, from about $635 \mathrm{~K}$ in the parent bismuth ferrite $(y=0)$ down to about $150 \mathrm{~K}$ in the composition with $y=0.60$ (Figure 3 ). The presence of the long-range antiferromagnetic ordering has also been confirmed by the neutron diffraction in the compositions with $y=0.3$ [19], $y=0.4$ (see Figure 4) and $y=0.5$ [20]. The $T_{\mathrm{N}}$ values were found to be practically the same for the non-annealed and annealed perovskites, independent on their crystal structure modification. The magnetization of the all studied perovskites is far from the saturation up to the highest applied field of $70 \mathrm{kOe}$.

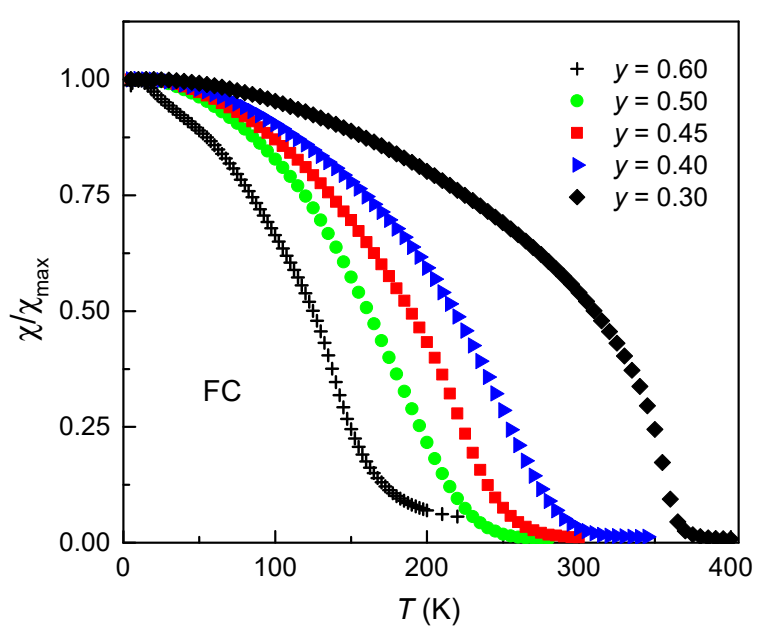

Figure 3. The normalized temperature dependent $d c$ magnetic susceptibility for the Pnma (I) polymorphs (as-prepared) of $\mathrm{BiFe}_{1-y} \mathrm{Sc}_{y} \mathrm{O}_{3}(0.30 \leq y \leq 0.60)$ measured in 50 Oe in the FC mode. 


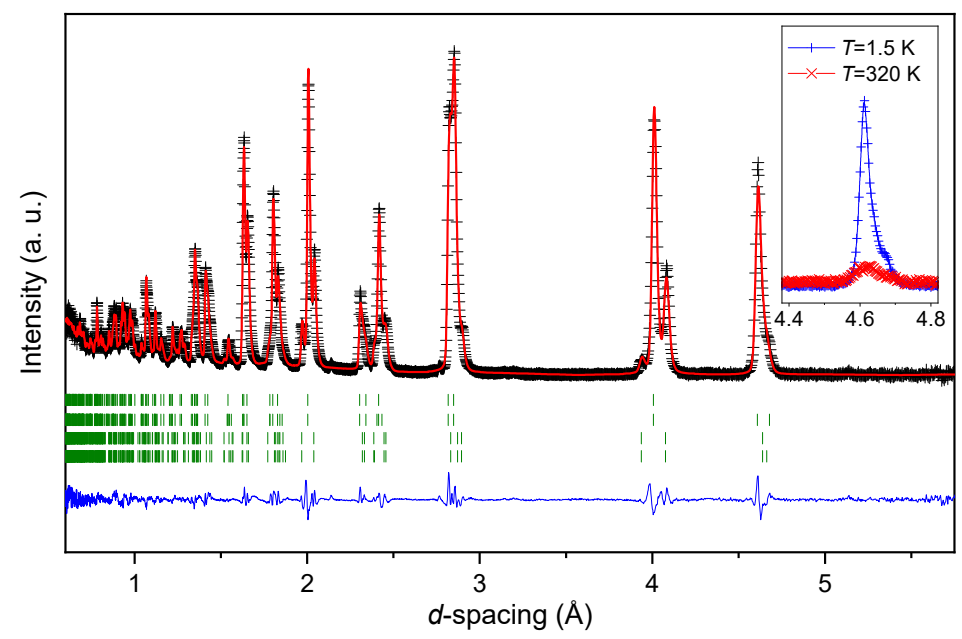

Figure 4. Neutron diffraction pattern of the annealed $\mathrm{BiFe}_{0.60} \mathrm{Sc}_{0.40} \mathrm{O}_{3}$ sample collected at $1.5 \mathrm{~K}$ and refined in the model contained two phases, namely the rhombohedral $R 3 c\left(70 \%, R_{B r a g g}=2.13 \%\right)$ and the orthorhombic Ima2 $\left(30 \%, R_{\text {Bragg }}=4.14 \%\right)$. The cross symbols and solid line (red) represent the experimental and calculated intensities, respectively, and the line below (blue) is the difference between them. Tick marks (green) indicate the positions of Bragg peaks (from top to bottom) for the nuclear rhombohedral, magnetic rhombohedral, nuclear orthorhombic and magnetic orthorhombic phases. Magnetic phases in both cases were G-type antiferromagnets (AFMs) with the moment direction along the $c$-axis in the rhombohedral phase $\left(\mathrm{R}_{\text {magnetic }}=3.12 \%\right)$ and along the $a$-axis in the orthorhombic phase $\left(R_{\text {magnetic }}=4.17 \%\right)$. Inset shows the diffraction patterns at the vicinity of the strongest magnetic peaks collected above and below $T_{\mathrm{N}}$.

In the $\mathrm{BiFe}_{1-y} \mathrm{Sc}_{y} \mathrm{O}_{3}$ solid solutions with the composition in the range of $0.20 \leq y \leq 0.60$, a weak FM component was detected. Figure 5 shows the static magnetic moment of a non-annealed $\mathrm{BiFe}_{0.70} \mathrm{Sc}_{0.30} \mathrm{O}_{3}$ sample, which is the orthorhombic Pnma (I) [19], as functions of temperature and field. It is seen from Figure $5 \mathrm{~b}$ that the orthorhombic polymorph of $\mathrm{BiFe}_{0.70} \mathrm{Sc}_{0.30} \mathrm{O}_{3}$ demonstrates weak ferromagnetism down to the low temperatures: its remnant magnetization and the coercive field are non-zero.
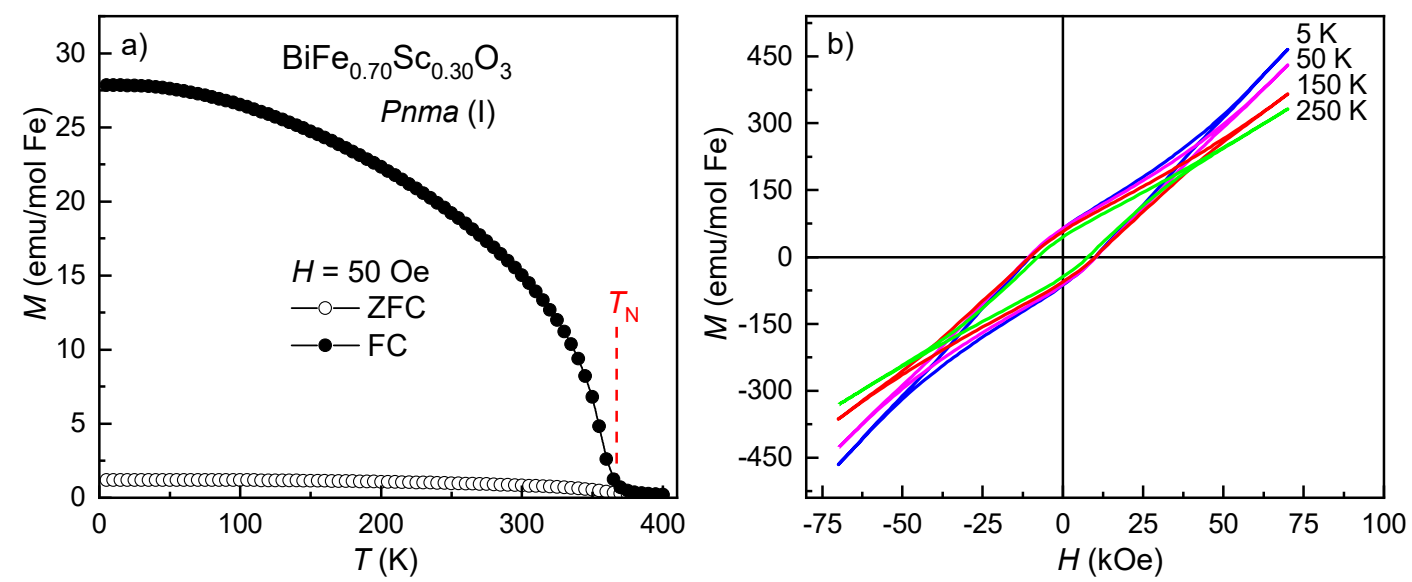

Figure 5. (a) Temperature dependent $d c$ magnetic moment for the as-prepared $\mathrm{BiFe}_{0.70} \mathrm{Sc}_{0.30} \mathrm{O}_{3}$ perovskite (the polymorph Pnma (I)) measured in 50 Oe in the ZFC (open symbols) and FC (solid symbols) modes. (b) Field dependent magnetization loops at different temperatures below $T_{N}$.

Annealing of the as-prepared metastable $\mathrm{BiFe}_{1-y} \mathrm{Sc}_{y} \mathrm{O}_{3}$ phases $(0.20 \leq y \leq 0.60)$ leads to the decrease of their magnetic moment in the magnetically ordered state, the remnant magnetization, and the coercive field. In other words, the annealing results in a reduction of the weak ferromagnetism and the magnetic anisotropy. It is known that annealing of the high-pressure synthesized ceramics is 
accompanied by a release of the mechanical stress induced by the quenching from high temperature and pressure down to ambient conditions [27]. In the samples of the $\mathrm{BiFe}_{1-y} \mathrm{Sc}_{y} \mathrm{O}_{3}$ system with $0.20 \leq y \leq 0.60$, a gradual stress release leads to the irreversible polymorphic transformations. [19].

Upon annealing, $\mathrm{BiFe}_{0.70} \mathrm{Sc}_{0.30} \mathrm{O}_{3}$ transforms into a rhombohedral $R 3 c$ polymorph, which is isostructural to the undoped bismuth ferrite. $\mathrm{BiFeO}_{3}$ is known to be $\mathrm{AFM}$ with an incommensurate cycloidal magnetic structure below $T_{\mathrm{N}} \sim 635 \mathrm{~K}$ [1]. The neutron diffraction study [19] revealed that the magnetic structure of the rhombohedral modification of $\mathrm{BiFe}_{0.70} \mathrm{Sc}_{0.30} \mathrm{O}_{3}$ just below $T_{\mathrm{N}} \sim 375 \mathrm{~K}$ is incommensurately modulated in the same manner as that in $\mathrm{BiFeO}_{3}$. However, while the cycloidal structure in bismuth ferrite remains upon cooling, the magnetic structure of $\mathrm{BiFe}_{0.70} \mathrm{Sc}_{0.30} \mathrm{O}_{3}$ below the transition temperature $T_{m} \sim 230 \mathrm{~K}$ becomes a collinear $G$-type AFM with the spins polarized along the threefold axis [19]. This structure allows no spin-canting and is the unique example of a $\mathrm{BiFeO}_{3}$-derived composition, in which the collinear magnetic structure does not activate the anti-symmetric exchange. In contrast with $\mathrm{BiFe}_{0.70} \mathrm{Sc}_{0.30} \mathrm{O}_{3}$, the rhombohedral phase of $\mathrm{BiFe}_{0.60} \mathrm{Sc}_{0.40} \mathrm{O}_{3}$ perovskite adopts the collinear AFM structure just below $T_{\mathrm{N}} \sim 300 \mathrm{~K}$ and this type of magnetic ordering remains stable over the whole temperature range (see Figure 4). The irreversible transformation of the $\mathrm{BiFe}_{0.60} \mathrm{Sc}_{0.40} \mathrm{O}_{3}$ composition into the $R 3 \mathrm{c}$ polymorph upon annealing was not complete and the annealed species contained $30 \%$ of the polar Ima 2 phase. The magnetic structure of the polar phase was found to be canted AFM similar to the Ima2 phase of $\mathrm{BiFe}_{0.50} \mathrm{Sc}_{0.50} \mathrm{O}_{3}$ [20].

Variations of the static magnetic moment of the rhombohedral polymorph of $\mathrm{BiFe}_{0.70} \mathrm{Sc}_{0.30} \mathrm{O}_{3}$ with temperature and the applied field are shown in Figure 6. The material demonstrates a usual AFM behaviour with a magnetization linearly dependent on the magnetic field (Figure 6b). One can notice two distinct regions in the temperature dependence of the magnetic moment below $T_{\mathrm{N}}$ (Figure 6a). (1) Between $T_{N}$ and $T_{m}$, the moment grows fast enough on cooling and a small difference between the ZFC and the FC curves is observed. (2) Below $T_{m}$, the moment changes slower and there is the evident near-constant difference between the ZFC and FC curves. Such a behavior is associated with a highly anisotropic state of the material below $T_{m}$. It should be also noticed that both magnetic transitions observed in the annealed $\mathrm{BiFe}_{0.70} \mathrm{Sc}_{0.30} \mathrm{O}_{3}$ at $T_{\mathrm{N}}$ and $T_{m}$ are both reversible with no visible temperature hysteresis.
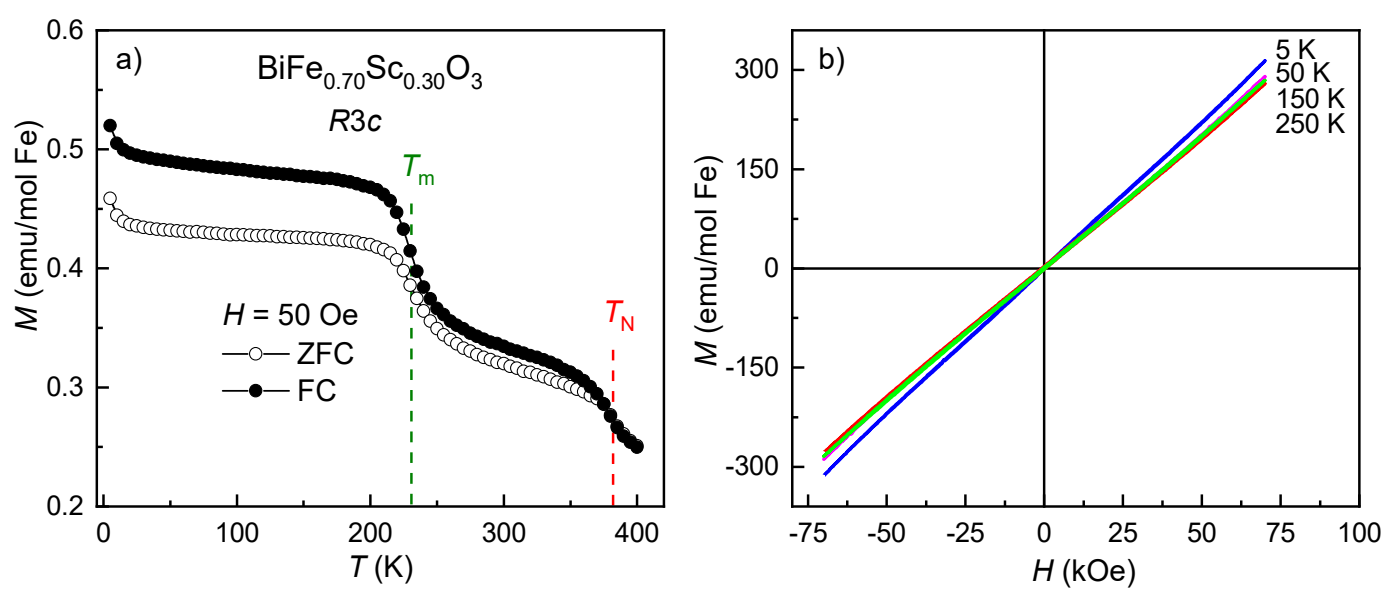

Figure 6. (a) Temperature-dependent $d c$ magnetic moment for the annealed $\mathrm{BiFe}_{0.70} \mathrm{Sc}_{0.30} \mathrm{O}_{3}$ perovskite (the polymorph $\mathrm{R} 3 \mathrm{c}$ ) measured in 50 Oe in the ZFC (open symbols) and FC (solid symbols) modes.

(b) Field dependent magnetization loops at different temperatures below $T_{\mathrm{N}}$.

Very similar temperature behavior of the static magnetic moment below $T_{\mathrm{N}} \sim 370 \mathrm{~K}$ was revealed in the annealed sample with $y=0.25$ (Figure 7). Following the description done for the temperature dependence of the magnetic moment of the $R 3$ c polymorph of $\mathrm{BiFe}_{0.70} \mathrm{Sc}_{0.30} \mathrm{O}_{3}$ (see above), the transition between two magnetically ordered states at $T_{m} \sim 175 \mathrm{~K}$ can be suggested. It is important to emphasize here that, as opposed to the $\mathrm{BiFe}_{1-y} \mathrm{Sc}_{y} \mathrm{O}_{3}$ composition with $y=0.30, \mathrm{BiFe}_{0.75} \mathrm{Sc}_{0.25} \mathrm{O}_{3}$ was found to 
exhibit no annealing-stimulated transformation [19]. The crystal structure of the latter remains the same, namely rhombohedral with the $R 3 c$ symmetry over the entire heating/cooling cycle. Nevertheless, the magnetic ground states of $\mathrm{BiFe}_{0.75} \mathrm{Sc}_{0.25} \mathrm{O}_{3}$ before and after annealing are different. This suggests that the phenomenon of conversion polymorphism is more general than considered previously: annealed-stimulated irreversible transformations of the magnetic structure are possible without change of the crystal structure, within the same structural polymorph.

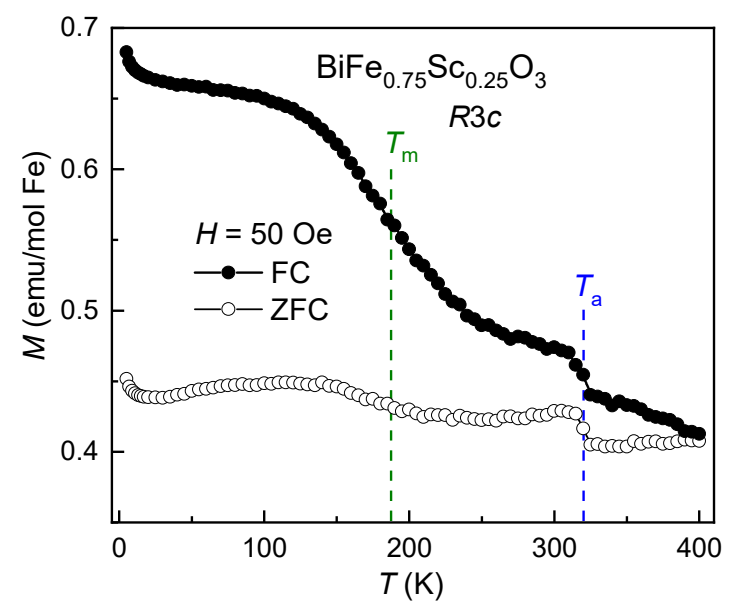

Figure 7. Temperature dependent $d c$ magnetic moment for the annealed $\mathrm{BiFe}_{0.75} \mathrm{Sc}_{0.25} \mathrm{O}_{3}$ perovskite (the polymorph R3c) measured in 50 Oe in in the ZFC (open symbols) and FC (solid symbols) modes.

The same type of the magnetic transformation was then found in other annealed $\mathrm{BiFe}_{1-y} \mathrm{Sc}_{y} \mathrm{O}_{3}$ perovskites with a lower content of scandium, namely those corresponded to $y=0.10$ and 0.20 (with $T_{m} \sim 105$ and $185 \mathrm{~K}$, respectively).

In addition to the magnetic phase transition at $T_{m}$, an anomaly in the temperature dependence of the magnetic moment, which indicates another magnetic transformation (at $T_{a}$ ), was observed in both as-prepared as well as annealed $\mathrm{BiFe}_{1-y} \mathrm{Sc}_{y} \mathrm{O}_{3}$ samples with scandium content in the range of $0.10 \leq y \leq 0.25$. The suggested magnetic transition at $T_{a}$ is strongly hysteretic, which implies that it is likely to be of the 1st order (Figure 7).

Based on the obtained data, a magnetic phase diagram of the $\mathrm{BiFe}_{1-y} \mathrm{Sc}_{y} \mathrm{O}_{3}$ system has been plotted (Figure 8).

There are at least three types of magnetically ordered structures in the system and all three are AFM. Two of those structures are the incommensurate cycloid and the collinear one as detected in the rhombohedral polymorph of $\mathrm{BiFe}_{0.70} \mathrm{Sc}_{0.30} \mathrm{O}_{3}$ above and below $T_{m}$, respectively. A question about a possible magnetic transition at $T_{a}$ in this polymorph remains open: whether it is very close to that at $T_{\mathrm{N}}$ or does not exist. The exact nature of the suggested AFM structures and transition between them in the range of $0.10 \leq y \leq 0.30$ certainly requires a particular study. Neutron diffraction experiments are planned and their results will be published elsewhere. 

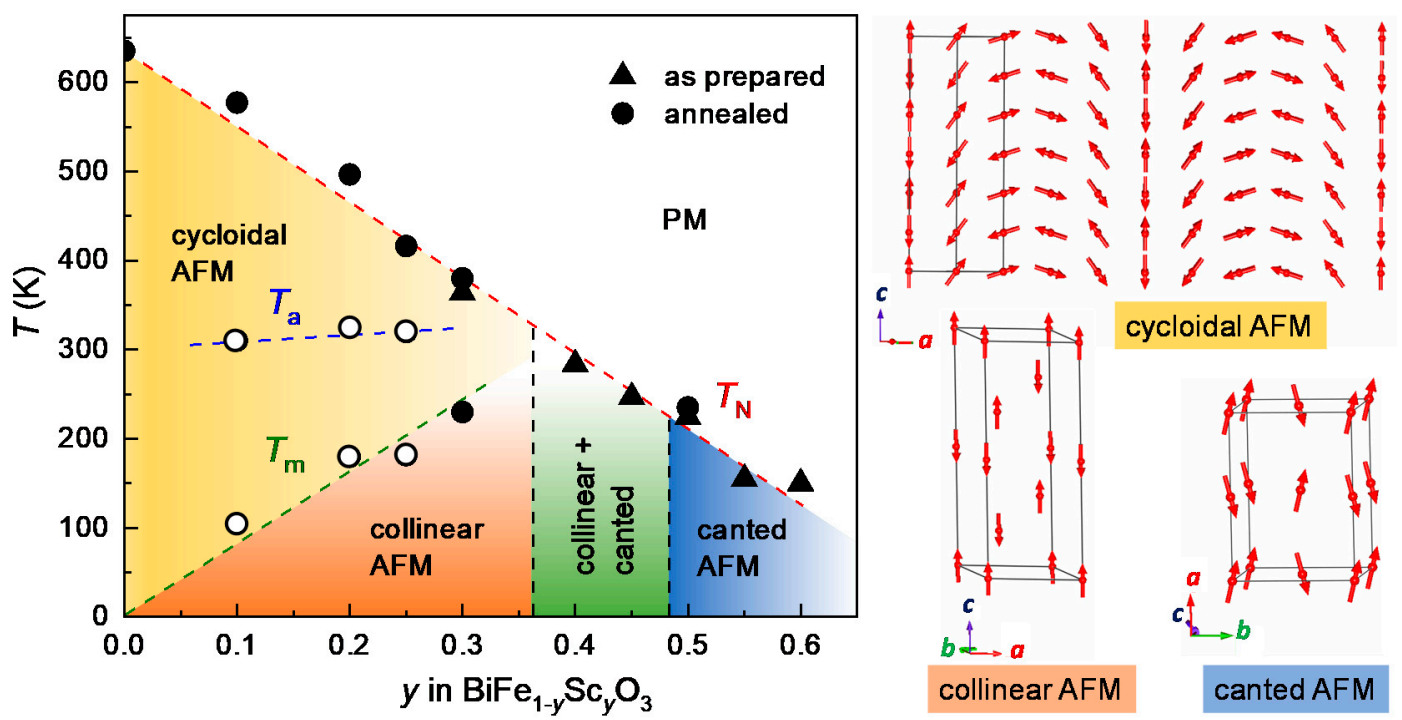

Figure 8. Magnetic phase diagram and schematic representation of the magnetic structures observed in the $\mathrm{BiFe}_{1-y} \mathrm{Sc}_{y} \mathrm{O}_{3}$ perovskites synthesized under high-pressure followed by annealing. The $T_{\mathrm{N}}$ value for the composition with $y=0$ was taken from Reference [1]. The lines between the points are guides to the eye. The phase transitions at $T_{\mathrm{m}}$ in the compositions with $y=0.1,0.2$ and 0.25 are less pronounced than in the $y=0.3$ sample, complicating the precise determination of their critical temperatures, and therefore the corresponding points are shown as open symbols. The area labelled as "collinear + canted" specifies the compositional-temperature range of two structural phases: the rhombohedral $R 3 c$ with the collinear AFM structure and the polar orthorhombic Ima2 with the canted AFM one.

\section{Conclusions}

The static magnetic moment of the as-prepared and the annealed $\mathrm{BiFe}_{1-y} \mathrm{Sc}_{y} \mathrm{O}_{3}$ solid solutions with the relative scandium content $y$ in the range of $0<y<1$ have been studied. Annealing of the as-prepared monoclinic $\mathrm{C} 2 / \mathrm{c}$ perovskite polymorph in the solutions with $y \geq 0.70$ results in the irreversible transformation (conversion polymorphism) to the antipolar orthorhombic Pnma (II) modification; nevertheless, the magnetic behaviors of both polymorphs are essentially similar: they are paramagnetic down to the low temperatures with no sign of magnetic ordering. The $\mathrm{BiFe}_{1-y} \mathrm{Sc}_{y} \mathrm{O}_{3}$ perovskites with $y \leq 0.60$ are all antiferromagnets with a near-linear dependence of $T_{N}$ on $y$. Although these perovskites in the range of $0.30 \leq y \leq 0.55$ exhibit the annealing-stimulated polymorphism, their $T_{\mathrm{N}}$ values are practically independent on the type of polymorph (before and after the annealing). In the perovskites with the composition in the range of $0.20 \leq y \leq 0.60$, a weak ferromagnetic contribution to the AFM state was detected. The annealing was found to lead to a reduction of this contribution.

In the $\mathrm{BiFe}_{1-y} \mathrm{Sc}_{y} \mathrm{O}_{3}$ solid solution with $y=0.30$, the conversion from the as-prepared antipolar orthorhombic Pnma (I) phase to the polar rhombohedral $R 3 c$ polymorph results in the appearance of a reversible phase transition (at $T_{m}<T_{\mathrm{N}}$ ) from the $\mathrm{BiFeO}_{3}$-like incommensurate cycloidal magnetic structure to a collinear G-type AFM with the spins polarized along the threefold axis. The solid solutions with a lower scandium content, $0.10 \leq y \leq 0.25$, exhibit no conversion polymorphism. Nevertheless, the annealed perovskites from this compositional range demonstrate the magnetic transition, which is very similar to that observed in the solid solution with $y=0.30$ at $T_{m}$. Moreover, the indications of another magnetic transition (at $T_{a}$, where $T_{m}<T_{a}<T_{\mathrm{N}}$ ) were observed in these solid solutions. The magnetic phase diagram suggests the presence of at least three different AFM structures in the $\mathrm{BiFe}_{1-y} \mathrm{Sc}_{y} \mathrm{O}_{3}$ system.

Author Contributions: Conceptualization, A.F., N.M.O. and A.R.B.; data curation, E.L.F., A.V.F., E.Č., S.V., Y.V.R., A.V.P., A.S. and D.D.K.; formal analysis, E.Č., N.M.O. and D.D.K.; investigation, E.L.F., A.V.F., E.Č., S.V., Y.V.R., A.V.P., A.S., D.D.K. and A.N.S.; methodology, N.M.O. and A.S.; project administration, A.F. and A.N.S.; resources, A.R.B.; supervision, A.F., A.R.B. and A.N.S.; validation, N.M.O.; visualization, A.V.F., D.D.K. and A.N.S.; 
writing—original draft, E.L.F.; writing—review and editing, D.D.K. and A.N.S. All authors have read and agreed to the published version of the manuscript.

Funding: The APC was funded by P.J. Šafárik University in Košice.

Acknowledgments: The authors acknowledge the financial support of the bilateral Slovakia-Belarus project "Novel environment-friendly materials for microelectronics based on complex bismuth-containing oxides with perovskite structure synthesized under high pressure" through grants APVV-SK-BY-RD-19-0008 and T20SLKG-001, respectively. Support of P.J. Šafárik University in Košice through grant VVGS-2020-1403 is also acknowledged. The research performed in the University of Aveiro was developed within the scope of the project CICECO-Aveiro Institute of Materials, UIDB/50011/2020 \& UIDP/50011/2020, financed by national funds through the FCT/MEC and when appropriate co-financed by FEDER under the PT2020 Partnership Agreement. Additional funding was provided by the Flexible Integrated Energy Systems (FLEXIS) operations funded by the Welsh European Funding Office (WEFO) through the Welsh Government.

Conflicts of Interest: The authors declare no conflict of interest.

\section{References}

1. Catalan, G.; Scott, J.F. Physics and Applications of Bismuth Ferrite. Adv. Mater. 2009, 21, $2463-2485$. [CrossRef]

2. Yang, C.-H.; Kan, D.; Takeuchi, I.; Nagarajan, V.; Seidel, J. Doping $\mathrm{BiFeO}_{3}$ : Approaches and enhanced functionality. Phys. Chem. Chem. Phys. 2012, 14, 15953-15962. [CrossRef]

3. Gebhardt, J.; Rappe, A.M. Doping of $\mathrm{BiFeO}_{3}$ : A comprehensive study on substitutional doping. Phys. Rev. $B$ 2018, 98, 125202. [CrossRef]

4. Arnold, D.C. Composition-driven structural phase transitions in rare-earth-doped $\mathrm{BiFeO}_{3}$ ceramics: A review. IEEE Trans. Ultrason. Ferroelectr. Freq. Control. 2015, 62, 62-82. [CrossRef] [PubMed]

5. Song, G.; Song, Y.; Su, J.; Song, X.; Zhang, N.; Wang, T.; Chang, F. Crystal structure refinement, ferroelectric and ferromagnetic properties of $\mathrm{Ho}^{3+}$ modified $\mathrm{BiFeO}_{3}$ multiferroic material. J. Alloys Compd. 2017, 696, 503-509. [CrossRef]

6. Surdu, V.-A.; Truşcă, R.; Vasile, B.S.; Oprea, O.; Tanasa, E.; Diamandescu, L.; Andronescu, E.; Ianculescu, A. $\mathrm{Bi}_{1-\mathrm{x}} \mathrm{Eu}_{\mathrm{x}} \mathrm{FeO}_{3}$ Powders: Synthesis, Characterization, Magnetic and Photoluminescence Properties. Nanomaterials 2019, 9, 1465. [CrossRef]

7. Belik, A.A.; Abakumov, A.M.; Tsirlin, A.A.; Hadermann, J.; Kim, J.; Van Tandeloo, G.; Takayama-Muromachi, E. ChemInform Abstract: Structure and Magnetic Properties of $\mathrm{BiFe}_{0.75} \mathrm{Mn}_{0.25} \mathrm{O}_{3}$ Perovskite Prepared at Ambient and High Pressure. Chem. Mater. 2011, 42, 4505-4514. [CrossRef]

8. Azuma, M.; Kanda, H.; Belik, A.A.; Shimakawa, Y.; Takano, M. Magnetic and structural properties of $\mathrm{BiFe}_{1-\mathrm{x}} \mathrm{Mn}_{\mathrm{x}} \mathrm{O}_{3}$. J. Magn. Magn. Mater. 2007, 310, 1177-1179. [CrossRef]

9. Mandal, P.; Sundaresan, A.; Rao, C.N.R.; Iyo, A.; Shirage, P.M.; Tanaka, Y.; Simon, C.; Pralong, V.; Lebedev, O.I.; Caignaert, V.; et al. Temperature-induced magnetization reversal in $\mathrm{BiFe}_{0.5} \mathrm{Mn}_{0.5} \mathrm{O}_{3}$ synthesized at high pressure. Phys. Rev. B 2010, 82, 100416. [CrossRef]

10. Azuma, M.; Niitaka, S.; Hayashi, N.; Oka, K.; Takano, M.; Funakubo, H.; Shimakawa, Y. Rhombohedral-Tetragonal Phase Boundary with High Curie Temperature in (1-x) $\mathrm{BiCoO}_{3-\mathrm{x}} \mathrm{BiFeO}_{3}$ Solid Solution. Jpn. J. Appl. Phys. 2008, 47, 7579-7581. [CrossRef]

11. Oka, K.; Koyama, T.; Ozaaki, T.; Mori, S.; Shimakawa, Y.; Azuma, M. Polarization Rotation in the Monoclinic Perovskite $\mathrm{BiCo}_{1-\mathrm{x}} \mathrm{Fe}_{\mathrm{x}} \mathrm{O}_{3}$. Angew. Chem. Int. Ed. 2012, 51, 7977-7980. [CrossRef]

12. Hojo, H.; Oka, K.; Shimizu, K.; Yamamoto, H.; Kawabe, R.; Azuma, M. Development of Bismuth Ferrite as a Piezoelectric and Multiferroic Material by Cobalt Substitution. Adv. Mater. 2018, 30, 1705665. [CrossRef] [PubMed]

13. Suchomel, M.R.; Thomas, C.I.; Allix, M.; Rosseinsky, M.J.; Fogg, A.M.; Thomas, M.F. High pressure bulk synthesis and characterization of the predicted multiferroic $\mathrm{Bi}\left(\mathrm{Fe}_{1 / 2} \mathrm{Cr}_{1 / 2}\right) \mathrm{O}_{3}$. Appl. Phys. Lett. 2007, 90, 112909. [CrossRef]

14. Raevski, I.P.; Kubrin, S.P.; Pushkarev, A.V.; Olekhnovich, N.M.; Radyush, Y.V.; Titov, V.V.; Malitskaya, M.A.; Raevskaya, S.I.; Chen, H. The effect of $\mathrm{Cr}$ substitution for Fe on the structure and magnetic properties of $\mathrm{BiFeO}_{3}$ multiferroic. Ferroelectrics 2018, 525, 1-10. [CrossRef]

15. Belik, A.A.; Rusakov, D.A.; Furubayashi, T.; Takayama-Muromachi, E. BiGaO 3 -Based Perovskites: A Large Family of Polar Materials. Chem. Mater. 2012, 24, 3056-3064. [CrossRef] 
16. A Belik, A. Magnetic properties of solid solutions between $\mathrm{BiCrO}_{3}$ and $\mathrm{BiGaO}_{3}$ with perovskite structures. Sci. Technol. Adv. Mater. 2015, 16, 026003. [CrossRef]

17. Pan, Z.; Chen, J.; Yu, R.; Yamamoto, H.; Rong, Y.; Hu, L.; Li, Q.; Lin, K.; You, L.; Zhao, K.; et al. Giant Polarization and High Temperature Monoclinic Phase in a Lead-Free Perovskite of $\mathrm{Bi}\left(\mathrm{Zn}_{0.5} \mathrm{Ti}_{0.5}\right) \mathrm{O}_{3}-\mathrm{BiFeO}_{3}$. Inorg. Chem. 2016, 55, 9513-9516. [CrossRef]

18. Salak, A.N.; Khalyavin, D.D.; Pushkarev, A.; Radyush, Y.V.; Olekhnovich, N.; Shilin, A.; Rubanik, V. Phase formation in the (1-y) $\mathrm{BiFeO}_{3-y} \mathrm{BiScO}_{3}$ system under ambient and high pressure. J. Solid State Chem. 2017, 247, 90-96. [CrossRef]

19. Khalyavin, D.D.; Salak, A.N.; Fertman, E.L.; Kotlyar, O.V.; Eardley, E.; Olekhnovich, N.M.; Pushkarev, A.V.; Radyush, Y.V.; Fedorchenko, A.; Desnenko, V.A.; et al. The phenomenon of conversion polymorphism in Bi-containing metastable perovskites. Chem. Commun. 2019, 55, 4683-4686. [CrossRef]

20. Khalyavin, D.D.; Salak, A.N.; Olekhnovich, N.M.; Pushkarev, A.V.; Radyush, Y.V.; Manuel, P.; Raevski, I.P.; Zheludkevich, M.L.; Ferreira, M.G.S. Polar and antipolar polymorphs of metastable perovskite $\mathrm{BiFe}_{0.5} \mathrm{Sc}_{0.5} \mathrm{O}_{3}$. Phys. Rev. B 2014, 89, 174414. [CrossRef]

21. Fedorchenko, A.; Fertman, E.L.; Salak, A.N.; Desnenko, V.; Čižmár, E.; Feher, A.; Vaisburd, A.; Olekhnovich, N.; Pushkarev, A.; Radyush, Y.V.; et al. Unusual magnetic properties of the polar orthorhombic $\mathrm{BiFe}_{0.5} \mathrm{Sc}_{0.5} \mathrm{O}_{3}$ perovskite. J. Magn. Magn. Mater. 2018, 465, 328-332. [CrossRef]

22. Fertman, E.L.; Fedorchenko, A.; Desnenko, V.A.; Shvartsman, V.V.; Lupascu, D.C.; Salamon, S.; Wende, H.; Vaisburd, A.I.; Stanulis, A.; Ramanauskas, R.; et al. Exchange bias effect in bulk multiferroic $\mathrm{BiFe}_{0.5} \mathrm{Sc}_{0.5} \mathrm{O}_{3}$. AIP Adv. 2020, 10, 045102. [CrossRef]

23. Khalyavin, D.D.; Salak, A.N.; Manuel, P.; Olekhnovich, N.M.; Pushkarev, A.V.; Radysh, Y.V.; Fedorchenko, A.; Fertman, E.L.; Desnenko, V.A.; Ferreira, M.G.S. Antisymmetric exchange in La-substituted $\mathrm{BiFe}_{0.5} \mathrm{Sc}_{0.5} \mathrm{O}_{3}$ system: Symmetry adapted distortion modes approach. Z. Krist.-Cryst. Mater. 2015, 230, 767-774. [CrossRef]

24. Chapon, L.C.; Manuel, P.; Radaelli, P.G.; Benson, C.; Perrott, L.; Ansell, S.; Rhodes, N.J.; Raspino, D.; Duxbury, D.; Spill, E.; et al. Wish: The New Powder and Single Crystal Magnetic Diffractometer on the Second Target Station. Neutron News 2011, 22, 22-25. [CrossRef]

25. Rodríguez-Carvajal, J. Recent advances in magnetic structure determination by neutron powder diffraction. Phys. B Condens. Matter 1993, 192, 55-69. [CrossRef]

26. Arévalo-López, A.M.; Alario-Franco, M.A. Structural Percolation in the $\mathrm{PbM}_{1-\mathrm{x}} \mathrm{M}_{\mathrm{x}}{ }^{\prime} \mathrm{O}_{3}\left(\mathrm{M}, \mathrm{M}^{\prime}=\mathrm{Ti}\right.$, Cr and V) Perovskites. Inorg. Chem. 2011, 50, 7136-7141. [CrossRef]

27. Khalyavin, D.D.; Salak, A.N.; Vyshatko, N.P.; Lopes, A.B.; Olekhnovich, N.M.; Pushkarev, A.V.; Maroz, I.I.; Radyush, Y.V. Crystal structure of metastable perovskite $\mathrm{Bi}\left(\mathrm{Mg}_{1 / 2} \mathrm{Ti}_{1 / 2}\right) \mathrm{O}_{3}$ : Bi-based structural analogue of antiferroelectric $\mathrm{PbZrO}_{3}$. Chem. Mater. 2006, 18, 5104-5110. [CrossRef]

Publisher's Note: MDPI stays neutral with regard to jurisdictional claims in published maps and institutional affiliations.

(C) 2020 by the authors. Licensee MDPI, Basel, Switzerland. This article is an open access article distributed under the terms and conditions of the Creative Commons Attribution (CC BY) license (http://creativecommons.org/licenses/by/4.0/). 